\title{
Inclusive websites for the elderly: user friendly guidelines for designers and managers of websites and applications
}

\author{
Alireza Darvishy ${ }^{1}$, Alice Good ${ }^{2}$ \\ ${ }^{1}$ School of Engineering, Steinberggasse 13, 8400. Winterthur \\ alireza.darvishy@zhaw.ch \\ ${ }^{2}$ University of Portsmouth, PO1 3AE \\ UK, alice.good@port.ac.uk
}

\begin{abstract}
This research aims to define the most significant criteria for guidance on accessible design. It highlights 9 significant areas of guidance which we consider to be very important for the design of accessible, elderly friendly websites and webapplications. These areas look at specific elements of web design, including: structure; navigation; language; multimedia; links; search as well as others. Each of these areas has defined checkpoints which can be used to test the accessibility of a website. The criteria and checkpoints are presented in the form of a user friendly brochure. This brochure is aimed at two target groups: an instrument for website managers and secondly a checklist for web designers.
\end{abstract}

Keywords: Accessibility, Elderly, Disability, Web design

\section{Introduction}

There is a definite need to 'bridge the gap' between elderly users and the Internet and with almost a fifth of the population aged over 65 , it is a significant market to exclude. The concern at the growing 'digital has led to a number of initiatives being employed to encourage web usage for the older generations and to ensure they are not excluded from the digital world. Many elderly people now make use of the Internet in controlling their finances, keeping up to date with current affairs and staying in contact with family and friends (DRC, 2004, Good, 200,). In considering that the elderly are very likely to develop age related impairments such as visual and/or mobility related disabilities, web accessibility then becomes an important consideration. However if web designers do not understand the guidelines that are put in place, then the problem of poor accessibility will inevitably continue

The focus of this paper is to present recommendations for the design and development of age appropriate websites. These recommendations form the basis of a 
brochure which presents are nine areas for web design tailored to senior citizens, each of which is dealt with on a double page. For each area, principles are formulated which are important for the design of websites tailored to senior citizens. The principles are primarily geared to clients, i.e. people who commission websites while web design and development specialists will also find specific instructions in the check lists regarding the implementation of the recommendations. The purpose of this booklet is to increasingly reduce the restrictions senior citizens face on a day-to-day basis when viewing not only residential and external areas on the web but also virtual areas.

\section{Methodology}

To be able to investigate the needs and requirements of the $65+$ generation with regard to user-friendly web design in greater detail, four focus groups with a total of 24 older participants, (average age 73 years), who use the Internet on a regular basis, were created in our project. The aim of the focus groups was to record the point of view of older web users as well as any obstacles that may arise which have been caused by the web design. An important result of the study revealed that the participating parties placed a great deal of importance on how well the web pages were designed. A few people reported that they were not able to use the websites in the way they wanted due to poor design and navigation.

Following the group interviews, the statements were compared with the latest specialist literature and discussed and extended by two circles of experts (specialists from the worlds of research, further education and senior citizens' organizations). Based on this multi-layered survey involving literature-based research, user focus groups and circles of experts, it was possible to formulate the recommendations outlined for "age-appropriate website design".

\section{Recommendations}

Nine areas for web design tailored to senior citizens are examined in greater detail below. Recommendations for implementation are also assigned to each area. The sequence of the areas is not relevant in itself, however, the discussions in the focus groups have shown that the first three of the following aspects were regarded in all groups as being very important:

Clarity and structure: The clarity and good structure of a website are important, if not in fact the most important prerequisites for achieving user-friendly web design. Senior citizens prefer information on a website to be ordered in as self-explanatory a way as possible, for the information to be restricted to what is absolutely essential and like having clear user guidance.

- The purpose of a website and its logical structure should be obvious the second a user looks at it. This means the various content, search and navigation areas must be well structured and their benefits or function must 
be self-explanatory. The selected layout must be applied as consistently as possible across all the web pages.

- If the content of the pages is arranged well, this makes the website easier to read and understand. Longer texts should be divided up in a sensible way. Related content areas can be linked together by navigational elements.

User guidance and navigation: User guidance which provides simple step sequences enables users to use the Internet on their own. Constructive and consistent navigation means older users feel they have adequate support

- Navigation elements must be self-explanatory and identifiable as such and must have the same design throughout the website.

- If icons or symbols are used for navigation purposes, their functions must be readily recognizable.

- Information about the user's current location on the website and the path the user took to get there help with orientation and contribute to effective user guidance.

Text and Language: The text is designed in such a way that it comprises a content component and a design component. The combination of both elements makes the site easier to read and understand. The design component also includes taking contrasts into account.

- Visitors to the website must be able to follow the information and understand it.

- With regard to the text design (line length, line spacing, spacing, contrasts), it is important to bear legibility and compatibility with different terminal devices in mind.

- Tools such as text enlargement are a good idea but should not impair the layout.

- Complementary (opposite each other in the chromatic circle) color combinations should be avoided.

Graphics, animated features and multimedia: Graphics and multi-media content are not only design elements, but are also used to display information and operate the web application. If they are reduced exclusively to their creative benefits, they can have a diverting influence or be very off-putting. The proportion of text to images should be balanced.

- As already discussed for text and language, there must be sufficient contrast with the images as well. From the point of view of readability and detectability, the size in which the images are displayed is important and it should be possible to adjust this size individually without any loss of quality.

- In the case of downloads, additional information regarding the content, the size as well as the loading times to be expected is helpful.

- Decorative elements such as animated images and background music often have a more jarring effect. 
Links: Links allow you to navigate within a website and to tap into additional as well as external information. If you are going to use links, make sure that they are clearly identifiable and self-explanatory.

- Links on a website should be clearly identifiable as such, also for visitors with restrictions such as color blindness and should be presented in a uniform manner throughout the entire website.

- Mouse-over effects (texts that are displayed as soon as the mouse pointer hovers over the link) have become an additional source of information for many users. However, these effects do not work with touchscreens (tablet PCs and smartphones) and for this reason you must provide alternatives.

Search: The search engine makes it easier to locate web content. Older people appreciate this function. They do, however, say that the manner of the search process is frequently not transparent and the order of the search results presented to them does not appear to be very logical. They have trouble, however, influencing this order.

- That the search fields are positioned well is just as important as the transparency of the search process. An option for refining the search through further criteria is welcomed.

- When the search results are displayed, the range of sorting options, for example, by date, place or other criteria can improve the overview.

Currentness, consistency and robustness: Older people appreciate it if the websites they use regularly as far as possible do not change their appearance (design) at all or only a little. They do, however, expect that the content is always up-to-date. Websites which are kept up-to-date usually provide a range of information of a very high standard. The structure and presentation of the entire website should therefore be as stable as possible. For users, finding their way on a newly designed page always involves a degree of learning. If the layout of web sites changes constantly, this reduces users' motivation to use the website.

- When it comes to layout, it is a balancing act between currentness and consistency. This, of course, does not apply to the content or the functionalities which must be kept up to date.

- The important thing is that the user quickly gets to grips with the website.

- It is important to note that the community of users is visiting the website from all kinds of terminal devices and the website should therefore be accessible on all such devices.

Contact information and support: In order to be able to support users of a website in the event of questions or problems, they must have some contact options. Here, in addition to web forms and e-mail, traditional contact options are also important for older people, namely the telephone or face-to-face contact at a counter. Older people also like to know who they are dealing with. For this reason, it is important to provide information about the provider of the website in the masthead. This helps to promote transparency.

- The contact information and the masthead should not just be available but should also be displayed in a prominent position on the site. 
- This also applies to other tools such as text enlargement, read-out function, glossary, FAQs, etc.

Registration and forms: Registrations and online forms require a self-explanatory design. The purpose and benefits of the data entry should make sense to the users immediately. With regard to data protection, data use, archiving and access by third parties must be able to be detected by users or explained on an additional page. For the design of online forms tailored to senior citizens, impaired sight and slower reaction times must also be taken into account.

- When personal data is entered, the meaning and purpose of the data and the necessary steps must be visible to the users before the data is input.

- As far as possible, incorrect entries should be eliminated when the data is first entered. In the event of errors, you should provide meaningful advice and a targeted correction function.

- What are known as captchas are often used to provide protection against spam. These, however, are difficult to read for older users and also for many people with "normal" vision. We recommend that you offer alternative security methods.

\section{Summary and Conclusions}

The recommendations presented in this document do not claim to be complete as the ever-changing Internet is constantly creating new challenges for older people in terms of accessibility. The recommendations and the checklist in this brochure can be used by clients and web developers alike. They provide clues for creating websites tailored to senior citizens. After a new website has been created, it should, however, also be checked and optimized using usability tests. Involving older users early on in the process can help identify and rectify specific problem areas in a website.

The premise of this paper is to present the principles for age appropriate design. The main brochure however proves full recommendations and guidance for the implementation of these guidelines. . It is anticipated that providing a user friendly brochure on the most prominent areas of accessible design guidance will inform designers better and subsequently lead to improved web accessibility.

\section{References}

1. Disability Rights Commission (2004). The Web: Access and Inclusion for Disabled People. London.

2. Good A., Stokes S. (2007). Elderly, Novice Users and Health Information Web Sites: Issues of Usability and Accessibility. Journal of Healthcare \& Information Management. Volume 21, Issue 1. 